\title{
Les activités transnationales des femmes immigrées. L'exemple d'une association de Marocaines de Bruxelles
}

Transnational Activities of Migrant Women. The Case of a Moroccan Association in Brussels

Las actividades transnacionales de las mujeres. El caso de una asociación de mujeres marroquíes en Bruselas

\section{Catherine Delcroix et Daniel Bertaux}

\section{(2) OpenEdition}

\section{Journals}

\section{Édition électronique}

URL : https://journals.openedition.org/remi/5779

DOI : $10.4000 /$ remi. 5779

ISSN : $1777-5418$

Éditeur

Université de Poitiers

\section{Édition imprimée}

Date de publication : 1 mars 2012

Pagination : 85-105

ISBN : 979-10-90426-03-0

ISSN : 0765-0752

\section{Référence électronique}

Catherine Delcroix et Daniel Bertaux, «Les activités transnationales des femmes immigrées. L'exemple d'une association de Marocaines de Bruxelles », Revue européenne des migrations internationales [En ligne], vol. 28 - $n^{\circ} 1$ | 2012, mis en ligne le 01 mars 2015, consulté le 14 avril 2022 URL : http://journals.openedition.org/remi/5779; DOI : https://doi.org/10.4000/remi.5779 


\section{Les activités transnationales des femmes immigrées. L'exemple d'une association de Marocaines de Bruxelles}

\section{Catherine DELCROIX ${ }^{1}$ et Daniel BERTAUX ${ }^{2}$}

\section{INTRODUCTION}

Qelon Rosita Fibbi et Gianni d'Amato, « la présence des femmes dans les

$\checkmark$ recherches sur le transnationalisme demeure extrêmement limitée (Pessar et Malher, 2003). (...) Il semble que l'invisibilité des femmes dans les études sur le transnationalisme tienne entre autres au fait que ces liens sont thématisés sous le terme de famille transnationale $(. .$.$) et ainsi en quelque sorte refoulés dans le domaine privé » (Fibbi et$ d'Amato, 2008 : 18). Le phénomène est-il quasi-inexistant? Ou s'agit-il seulement d'une absence de recherches empiriques?

Nous avons voulu en avoir le cœur net et nous avons entamé une recherche sur les (hypothétiques) activités transnationales des Marocaines de Bruxelles. Nous dirons ci-après les raisons de ce choix ; et comment, dès les premiers mois d'enquête, nous en avons découvert une grande variété, portées par des associations. L'essentiel de cet article est constitué par l'étude de cas des activités transnationales de l'une d'elles.

À vrai dire, nous avions déjà découvert, il y a une quinzaine d'années, au cours d'une enquête sur la formation et l'action des médiatrices socioculturelles effectuée dans toute la France (vingt-deux sites d'observation) des exemples de ce phénomène. Dans leur grande majorité, les médiatrices de quartiers défavorisés que nous avions alors rencontrées étaient des immigrées. Presque toutes avaient commencé en regroupant autour d'elles d'autres immigrées de leur immeuble, de leur rue, de leur quartier. Les plus dynamiques

1 Professeure de sociologie à l'Université de Strasbourg, Laboratoire « Cultures et Sociétés en Europe »; catherine.delcroix@misha.fr

2 Directeur de recherches émérite au CNRS ; daniel.bertaux@misha.fr 
avaient fini par fonder une association; et plusieurs de ces associations menaient des actions envers tel ou tel pays du Maghreb (Delcroix et al., 1996).

Après cette expérience, nous voulions de longue date étudier ce type d'activités plus en détail. Nous avons choisi de partir des associations de Marocaines de Bruxelles. $13 \%$ de la population de Bruxelles (130 000 personnes sur 1 million) est d'origine marocaine, cas unique en Europe. De plus, deux « modèles d'intégration » très différents y coexistent côte à côte : le flamand et le francophone (Adam, 2010). Cela rend la situation de Bruxelles quasi-expérimentale.

Nous allons tout d'abord revenir brièvement sur le concept de transnationalisme. Nous présenterons ensuite les Marocain(e)s de Bruxelles et les deux modèles d'intégration présents dans cette ville. Dans un troisième temps, dans la partie principale, seront analysées les activités transnationales d'une association, sa genèse et cinq projets. À la fin sera proposée une discussion sur les éléments de portée générale que cette étude de cas permet de dégager.

\section{TRANSNATIONALISME ET ACTIVITÉS TRANSNATIONALES}

Le terme de transnationalisme a suscité nombre de débats théoriques au sein de la sociologie anglo-saxonne de l'immigration. Sa signification a fini par se stabiliser. Fibbi et d'Amato (2008 : 7) le définissent ainsi : «Par transnationalisme on entend une construction de champs sociaux dans laquelle les migrants créent un lien imaginaire ou réel entre leur pays d'origine et leur société d'accueil (Glick-Schiller, Basch et SzantonBlanc, 1992 ; Portes, 1997). Dans ces espaces transnationaux, les migrants mettent en place des relations sociales et économiques, des activités et des identités politiques qui transcendent les frontières classiques (...). Le transnationalisme représente un déplacement des identités notamment nationales et des revendications politiques et sociales au-delà des appartenances territoriales habituelles fondées sur l'échelle des États-Nations (...). Ainsi l'approche du transnationalisme a-t-elle pu se positionner dans le champ de la migration comme une sorte de troisième voie entre l'option de l'assimilation et celle du retour; entre une perspective où l'action individuelle est surdéterminée par les contraintes macro sociales (Portes, 1999) et une perspective qui conçoit celle-ci comme le résultat de la volonté et des préférences des acteurs sociaux $»$.

Quant aux activités transnationales, il s'agit des « activités déployées par les migrants impliquant au moins deux pays, incluant des activités entre le pays de résidence et le pays d'origine et/ou des ancêtres, ainsi que les activités menées dans des pays tiers, quel que soit le degré d'implication des co-ethniques » (Fibbi et d'Amato, 2008 : 8). Les recherches sur ce thème se sont concentrées jusqu'ici sur trois sphères d'activités, l'économique, le socioculturel et le politique.

Cependant ces activités n'ont été conçues jusqu'à présent que comme exclusivement portées par des hommes. Or l'enquête nationale sur les médiatrices de quartier mentionnée plus haut, ainsi que d'autres travaux plus récents (Delcroix, 2007, 2010a ; Veith, 2005 ; Lacroix, Sall et Salzbrunn, 2008) montrent que beaucoup de femmes 
immigrées, loin d'avoir coupé les ponts avec leur pays d'origine, y développent des activités bénévoles ou lucratives.

\section{BRUXELLES : LES MAROCAIN(E)S ET LES MODÈLES D'INTÉ- GRATION}

On estime à 160000 les Belges d'origine marocaine et à 80000 les Marocain(e) s qui vivent en Belgique ; soit au total 240 000, ou 2,4\% de la population (10 millions d'habitants). Ils constituent l'une des « minorités culturelles » les plus nombreuses de Belgique, la plus nombreuse après les Italiens (Gaudier et Hermans, 1991). Plus de la moitié d'entre eux, 130 000, vivent à Bruxelles. Dans certains quartiers du centre-ville, ils constituent jusqu'à $40 \%$ de la population.

Contrairement à la France, la Belgique ${ }^{3}$ compte un grand nombre d'associations spécifiquement marocaines. Rien qu'à Bruxelles, on en dénombre soixante-dix-neuf, dont presque la moitié $(\mathrm{n}=37)$ sont dirigées à parité ou majoritairement par des Marocaines ${ }^{4}$. Les Marocains de Belgique proviennent surtout du nord du Maroc, des zones rurales du Rif et de grandes villes comme Tanger, Tétouan et Oujda. Ceux qui viennent du Rif parlent le berbère ; les autres sont arabophones. La première vague d'immigration marocaine est arrivée dans les années 1960, quand « le marché ouest-européen de l'emploi s'est largement ouvert à l'immigration d'ouvriers non-qualifiés » (Rea, 2003 : 214). Le Maroc venait d'obtenir sa pleine indépendance et son gouvernement a signé des accords avec plusieurs gouvernements européens dont les firmes industrielles et du bâtiment recherchaient de la main-d'œuvre bon marché. En 1964 par exemple, ce sont 90000 jeunes Marocains qui sont venus en Belgique pour y travailler (Gaudier et Hermans, 1991).

Dès 1956, les autorités belges, pour attirer les « travailleurs immigrés », avaient autorisé l'installation de leurs conjoints et enfants. L'immigration marocaine est donc passée très vite d'une immigration de travail à une immigration de peuplement. Actuellement, les flux d'immigration sont à nouveau en augmentation : le solde migratoire avec le Maroc est de plus de 30000 personnes par an, « soit presque autant que dans la période 1960-64, les années phares de l'histoire de l'immigration en Belgique au XXe siècle » (Eggerickx et al., 2010). Quant aux naturalisations, 150000 étrangers sont devenus belges entre 1988 et 2002, dont la moitié des Marocains et Turcs de Bruxelles.

La Belgique est divisée en trois grandes régions : la Flandre, la Wallonie, et la région Bruxelles-capitale. Or les politiques dites en Belgique « d'accueil et d'intégration des immigrés » sont différentes en Flandre et en Wallonie ; et dans la Région Bruxellescapitale elles sont toutes deux présentes... et en concurrence l'une avec l'autre.

$3 \mathrm{La}$ Belgique est un pays multiculturel depuis sa fondation en 1830 par l'union de deux peuples catholiques, les Wallons francophones et les Flamands néerlandophones, qui refusaient la domination de la famille royale hollandaise (et protestante).

4 Selon l'enquête en cours sur les associations d'immigrés de Bruxelles effectuée par Rebecca Thys, doctorante de Dirk Jacobs, professeur à l'ULB et codirecteur du GERME. 
En Wallonie et dans la partie francophone de Bruxelles la spécificité culturelle des immigrés n'est pas reconnue. Leurs associations ne peuvent donc revendiquer des subventions à ce titre. Les politiques sociales se sont longtemps adressées à eux en tant $q u$ 'ouvriers, sans distinction avec les ouvriers wallons ; aujourd'hui, c'est plutôt, signe des temps, en tant que « publics défavorisés » (Rea, 2003). Les mots-clés sont ceux d'intégration sociale et de cohabitation.

En Flandre par contraste, les communautés immigrées ont été rebaptisées «minorités ethnoculturelles »; leur spécificité culturelle est pleinement reconnue et les organisations autonomes de ces « communautés » sont chargées de la préserver.

La situation à Bruxelles est donc fort complexe et en pleine évolution dynamique. La capitale du pays est géographiquement située en Flandre, et donc revendiquée en tant que telle par les Flamands ; mais $85 \%$ de sa population de 1 million d'habitants est francophone.

Multiculturalisme du côté flamand, « assimilationnisme » du côté francophone s'y font face, dans des luttes d'influence de plus en plus vives pour s'attirer la sympathie des dites " communautés immigrées » extra-communautaires. Et surtout leurs votes, puisqu'elles ont obtenu en 2006 (avec le soutien de tous les partis francophones et contre presque tous les partis flamands) le droit de vote aux élections locales. Depuis 2006 leurs votes ont fait basculer Bruxelles à gauche (Rea, Jacobs et Terney, 2010).

Les stratégies sont différentes. La Flandre est plus prospère que la Wallonie, et l'argent flamand est investi à Bruxelles dans la construction de nouvelles écoles néerlandophones $^{5}$. Les enfants d'immigrés marocains et turcs y sont les bienvenus, pour autant qu'ils acceptent d'apprendre et de parler le flamand : on dit aux parents qu'ainsi leur avenir professionnel sera meilleur. Venus de Flandre, des hommes politiques, des enseignants, des animateurs socioculturels viennent continûment s'installer à Bruxelles.

Du côté francophone on procède différemment. Les fonds de la Politique de la Ville initiée en 1993 ont été largement consacrés à l'embauche de travailleurs sociaux et " médiateurs » d'origine immigrée, qui ont été très vite cooptés comme " représentants de la diversité » dans les partis politiques francophones. Les associations d'immigrés sont financées si elles acceptent de travailler avec eux, et de diffuser les informations sur les politiques publiques francophones.

La vive concurrence entre Flamands et Francophones à Bruxelles crée des opportunités politiques pour les minorités immigrées. Ce n'est sans doute pas un hasard si les dirigeantes de trois des plus grosses associations de Marocaines de Bruxelles engagées dans des activités transnationales nous ont dit qu'elles avaient été assistantes parlementaires auprès de politiciens de différents partis francophones ou flamands ${ }^{6}$. Cependant

5 Par contraste, du côté francophone on constate une dégradation générale des conditions d'enseignement : baisse des salaires des enseignants, augmentation du nombre d'enfants par classe, vieillissement des locaux, etc.

6 Martiniello et Boussetta (2008) avaient déjà remarqué que tous ceux des immigrés (masculins) marocains ou chinois de Belgique qui s'engageaient dans des activités transnationales jouissaient déjà auparavant d'une certaine notabilité. 
toutes trois affirmaient se distancier des jeux politiques dans leurs engagements transnationaux et chercher à « faire de la politique autrement ».

\section{LES ACTIVITÉS TRANSNATIONALES D'UNE ASSOCIATION DE FEMMES}

Dès le début de notre enquête de terrain, nous avons rencontré la femme médecin qui dirige une Maison Médicale du quartier de Schaerbeek, l'une des trois " communes » de Bruxelles (l'équivalent des arrondissements de Paris) avec Mollenbeek et Forest qui comptent la plus forte proportion de personnes d'origine marocaine. Elle y travaille depuis trente ans : " 3500 personnes y sont actuellement soignées "au forfait", dont $75 \%$ de femmes, et $90 \%$ d'immigré(e)s ». Elle y a fait un grand nombre d'accouchements et de suivis gynécologiques d'immigrées, avec lesquelles elle a ainsi créé des liens de confiance très forts dans la longue durée. Nous cherchions des exemples de Marocaines ayant des activités avec le Maroc : "Oh j'en connais plein!", s'est-elle écriée, ajoutant aussitôt qu'elle était frappée par la rapidité avec laquelle ces femmes se mobilisent, individuellement et surtout « en réseau » : "Mais il faut qu'il y ait un objectif concret; si c'est pour écouter un philosophe, non elles ne viennent pas. Il faut qu'il y ait des besoins concrets, qu'elles peuvent aider à satisfaire ».

Grâce à elle nous avons pu très rapidement entrer en relation avec nombre de Marocaines très actives dans la vie associative. Parmi celles-ci il y avait Najat, présidente de l'association Dar el Ward (La Maison des fleurs). Une de ses activités à Bruxelles consiste à préparer, deux fois par semaine, 100 à 150 repas pour les sans-domicile-fixe, les sans-papiers, les gens en prison. Ces repas sont ensuite distribués non seulement à Bruxelles mais en divers endroits de Belgique, à tour de rôle, par un réseau d'une soixantaine de jeunes, filles et garçons, d'origine marocaine ${ }^{7}$. À l'occasion de la fête de l'Aïd qui clôt la période du Ramadan nous avons participé, toute une journée, à cette activité avec dix femmes de l'association; et c'est en parlant avec elles que nous avons commencé à découvrir l'étendue de leurs activités au Maroc qui s'incarne dans des projets.

Peu après, Najat nous a proposé de l'accompagner au Maroc dans une tournée qu'elle comptait effectuer concernant certains de ces projets. Nous avons accepté l'invitation; et ce voyage d'ethnographie multi-sites a été une révélation. Nous ne pouvions imaginer meilleur démenti de l'inexistence supposée des activités transnationales des immigrées. Compte tenu de l'écart entre représentations a priori et réalités du terrain, il nous a paru nécessaire d'en rendre compte dès que possible. Nous présenterons donc ici les résultats de ce «terrain » sous la forme d'une étude de cas inspirée de la démarche de Geertz (1973).

\section{Genèse}

L'initiative de cette association est venue en 1991 d'une quinzaine de Marocaines de Schaerbeek. Elles avaient à l'époque entre vingt-cinq et trente ans, elles étaient déjà

7 Chaque semaine une dizaine d'entre eux sont mobilisés. 
mariées et ne se contentaient pas de rester au foyer. Najat, qui avait un bon emploi et se retrouvait à trente ans divorcée avec deux enfants, leur proposa d'organiser des activités communes. Elle faisait déjà beaucoup de bénévolat ${ }^{8}$.

Ces activités prennent assez rapidement de l'ampleur :

"Par exemple on organisait des sorties dans toutes les villes de Belgique. Pendant les congés scolaires, on a décidé d'organiser des camps pour les femmes et les enfants. On a travaillé cinq ou six ans rien qu'entre nous, entre femmes. Et puis on a décidé de partir quinze jours au Maroc ».

Le groupe en reste au stade informel de réseau d'amies jusqu'en 1999, date à laquelle l'association est créée sous la forme d'une ASBL (Association Sans But Lucratif). Elles la baptisent Dar el Ward, « La maison des fleurs ».

«Et puis on a décidé de partir quinze jours au Maroc et de louer des cars. C'est là qu'on a commencé à prendre des hommes avec nous. On sélectionnait des hommes assez ouverts et constructifs. On en a eu qui se plaignaient qu'ils ne

voyaient plus leur femme, qu'ils ne contrôlaient plus leur fille, et on leur disait qu'on avait vraiment besoin d'eux. Nous avions besoin que les hommes permettent aux femmes d'aller plus loin dans leur activité ; et pour cela il fallait garder leur confiance $e^{9}$. On avait décidé de faire du tourisme pendant une semaine, et de l'humanitaire pendant l'autre semaine. Depuis cette période on a fait plus de vingt voyages. À chaque fois dans une nouvelle région du Maroc; en passant toujours par

un projet qu'on avait initié auparavant : un orphelinat, une maison de repos pour personnes âgées, un projet d'accueil pour des enfants atteints du cancer, pour mieux les soigner...».

8 Née en 1961, la cinquième d'une fratrie de huit, elle était arrivée en Belgique à quatre ans avec sa mère et ses frères et sœurs. Son père y travaillait déjà comme cuisinier dans un grand restaurant. Elle a grandi à Hœillart, une commune flamande de la région bruxelloise. Sa mère était très investie dans l'accueil et le soutien informel aux primo-arrivants du Maroc. Après avoir réussi des études secondaires dans la « spécialité commerce » Najat se marie en 1982 (ses deux enfants naîtront en 1985 et 1987) mais est déterminée à ne pas lâcher sa carrière. Elle a été engagée en 1981 dans une entreprise d'import-export, où elle a commencé à l'accueil mais dont elle gravira rapidement les échelons, devenant cadre avec un bon revenu mensuel. Elle est restée dans cette entreprise jusqu'en 2001 (entretemps, en 1989, elle a divorcé). Entre 2003 et 2004 elle travaille dans une agence de coopération où elle est chargée d'aider des Marocains de Belgique, désireux de rentrer au pays, à concevoir des projets de développement au Maroc. Elle a été aussi collaboratrice d'un député flamand et conseillère du ministre de la Culture dans la cellule diversité.

9 Si le groupe s'ouvre aux « hommes » : maris, pères, etc., il est cependant clair que le noyau des fondatrices de l'association entend bien en garder le contrôle. Car, précisera Najat, « les hommes ont tendance à prendre le côté facile. Ils n'accompagnent pas un projet concret, ils ne le portent pas avec leurs tripes, leurs bras, et leur poche !... Par exemple, on a un convoi humanitaire : on trie, on reçoit, on emballe, on recherche les fonds pour acheminer les dons jusqu'à leur destination finale. L'homme, même s'il n'a apporté qu'un petit carton, il va venir avec son appareil photo : il va vouloir le montrer $»$. 
Ces trois projets - l'association en porte au moins une quinzaine d'autres - constituent des exemples d'activités transnationales. Tous sont d'ordre humanitaire ; mais il nous apparaîtra peu à peu qu'ils ont d'autres points communs qui, pris ensemble, dessinent une « forme », une configuration stable et récurrente.

\section{Cinq projets au Maroc}

C'est grâce aux liens de confiance qui s'étaient tissés au fil des rencontres à Bruxelles entre les dirigeantes de l'association et nous-mêmes (voir Delcroix, 2010b) que Najat nous a proposé d'aller avec elle au Maroc. Elle devait y faire la « tournée » de huit des vingt projets que l'association y soutient, souvent en partenariat avec d'autres associations - belges, marocaines, ou internationales - ou des institutions marocaines. Tel qu'elle l'avait planifié, le voyage nous mènerait de Tanger à Tétouan, puis dans deux villages du Haut-Atlas, ensuite à Rabat et enfin à Casablanca. Bien que ce voyage n'ait duré que deux semaines, nous y avons beaucoup appris. Nous avons pu longuement nous entretenir avec les responsables de chacun des huit projets visités et avec certains de leurs usagers. À Rabat, nous avons rencontré des personnages influents. Au-delà de la connaissance et de la compréhension du fonctionnement de tel ou tel projet, des nombreux obstacles affrontés et des façons d'y faire face, nous avons commencé à acquérir une vision d'ensemble des multiples cours d'action engagés par l'association au Maroc ; et à saisir, par-delà leur diversité, ce qui pouvait en faire l'unité.

\section{L'association « La Colombe Blanche » pour la protection des jeunes handi- capés}

Après avoir atterri à Tanger, nous sommes allées directement à Tétouan. C'est la ville d'origine des parents de Najat ; et c'est là aussi que le groupe des femmes fondatrices de Dar el Ward a initié depuis 1996 plusieurs projets qui sont toujours opérationnels aujourd'hui.

Le premier des projets que Najat voulait me montrer était « La Colombe Blanche ». C'est une association qui se bat pour que les handicapés physiques aient accès à leurs droits de citoyens marocains. La genèse de cette association, l'histoire de l'aide qu'elle a reçue de Dar El Ward, et le parcours exceptionnel de son fondateur Abdelmalek A. forment un trio inséparable.

Commençons par Abdelmalek A. Il naît dans un village de montagne du Rif, le quatorzième enfant d'une famille qui en compte vingt. Les revenus de la famille sont faibles. Il n'a que quatorze mois quand son handicap se révèle : l'une de ses jambes est très faible. Quand viendra le temps d'aller à l'école, il lui faudra des béquilles ; ses frères lui en fabriquent avec du bois. Mais comment faire pour rejoindre le car qui emmène les enfants du village jusqu'à l'école ? Toute la famille se mobilise pour l'aider à être scolarisé. Un frère aîné qui a déjà un emploi d'infirmier le prend plus particulièrement en charge, lui apportant tout au long de sa scolarité un soutien moral et financier constant. À neuf ans il est enfin scolarisé à l'école primaire où vont tous les autres enfants. 
À partir de ce moment-là, il fait tout son possible pour figurer parmi les meilleurs de la classe, et y parvient. Tout au long de sa scolarité, il renouvellera des demandes de bourse, toujours rejetées. Il réussit cependant à terminer ses études secondaires ; puis à s'inscrire à l'Université, en Droit (on comprend pourquoi). Surmontant de nombreuses privations il obtient son diplôme universitaire. Il cherche alors un emploi, mais n'en trouve pas. Chômeur sans revenu pendant six ans il poursuit cependant inlassablement de multiples activités bénévoles. Il devient Président de l'Association des anciens étudiants en droit de l'université de Tétouan. En 1993, avec quelques amis - garçons et filles à mobilité réduite comme lui, il fonde l'association La Colombe pour la protection des jeunes handicapés. Deux des fondateurs sont des jeunes de niveau universitaire, trois ont un niveau bac, deux sont sans diplôme. Ces sept jeunes voudraient que la gestion des handicaps physiques se fasse autrement au Maroc. Leur projet est cohérent, mais ils se heurtent d'emblée à de gros obstacles. Ahmed A., l'actuel Président de La Colombe (qui s'est considérablement développée depuis) explique lesquels :

"À l'époque de la création de l'association sur Tétouan, il existait déjà une association, HANANE, soutenue et portée par les notables de la ville. Ils préconisaient pour les enfants handicapés une éducation spécialisée en milieu protégé. Nous, nous avions un tout autre esprit : nous venions de familles qui s'opposaient aux préjugés contre les handicapés. Nous avions suivi une scolarité dans des écoles ordinaires. Nous voulions faire accepter cette approche et l'associer à une lutte plus large pour les droits de l'homme en général, pour une intégration sociale équitable. Ce que nous avons fait, c'est agir en suivant quatre axes: 1. Sensibiliser pour une place de notre mouvement dans la société civile ; 2. travailler avec la presse pour montrer notre valeur ajoutée par rapport à d'autres associations existantes. 3. Dès le début nous avons visé les acteurs politiques et ceux de la société civile. Et 4. nous

avons orienté notre militance sur toutes les causes : femmes, enfants, environnement, démocratie locale. À partir de cette approche nous avons construit notre place comme acteur incontournable dans le domaine du handicap ».

Tout ce temps-là, la situation financière d'Abdelmalek est extrêmement précaire. La rencontre en 1996 avec Najat. S. s'avèrera décisive. Najat :

"Le lien entre Dar El Ward et la Colombe Blanche s'est construit lors d'une action humanitaire organisée par Dar el Ward au profit des personnes en situation précaire à Tétouan. J'ai eu l'occasion de faire la connaissance d'Abdelmalek A., qui était président de l'association La Colombe. L'association a été bénéficiaire parmi d'autres du convoi composé de chaises roulantes, béquilles, vêtements, jouets, matériels scolaires, etc. J'ai alors réalisé qu'Abdelmalek avait beaucoup de compétences, qui restaient non exploitées par manque de moyens matériels et financiers. À partir de ce constat, nous avons décidé en 1996 d'un commun accord de la mise en place d'une petite structure composée de cinq personnes : il s'agissait d'un appartement qui servait à la fois de bureau et de logement pour Abdelmalek. Il était 
équipé très simplement : un bureau, quelques chaises, un fax et un ordinateur. Cette structure a été mise en place par mes fonds propres $»^{10}$.

N'ayant plus à se préoccuper de payer son loyer, Abdelmalek peut enfin se concentrer sur la préparation d'un concours administratif qu'il réussit et il est recruté par la municipalité de Tétouan comme agent administratif. Cette embauche ne signifie pas seulement sa sortie de la précarité, mais aussi la mise en contact quotidienne avec des élus, des notables et le début d'une brillante carrière qui le conduira, en tant que militant et représentant des handicapés moteurs, jusqu'à la capitale Rabat et même aux Nations Unies $^{11}$.

Pendant plus de vingt ans, entre 1996 et 2008, date à laquelle il partira pour la capitale, Abdelmalek A. sera le très efficace, fidèle et créatif correspondant de l'association Dar el Ward à Tétouan. Il développera des projets sur le handicap non seulement à Tétouan mais dans tout le Maroc et suivra aussi une série d'autres projets, mettant en rapport entre eux leurs divers responsables. Il a gardé une attitude militante et partage entièrement l'esprit de l'association Dar el Ward, qu'il exprime à sa façon :

«L'important c'est la philosophie du social : ce n'est pas par la charité qu'on règlera les questions ; c'est par l'équité et la participation de la population ».

L'exemple de la coopération entre Dar el Ward et La Colombe est significatif du fonctionnement de ces activités transnationales. L'une de ses membres (dans ce cas-ci c'est la présidente) identifie dans une ville (ici Tétouan) un manque matériel (ici des fauteuils roulants) derrière lequel se trouve l'absence d'une politique publique de prise en charge

10 Najat a toujours participé financièrement aux projets de l'association, même si sa première expérience a été un cuisant échec : «Je voudrais te raconter l'échec d'une action qui (finalement) m'a renforcée, et qui m'a permis ensuite de rencontrer Abdelmalek. En 1995 j'avais rencontré (à Bruxelles) un homme (d'origine marocaine) engagé politiquement pour un parti francophone. Il était le collaborateur d'un parlementaire dont j'avais fait la campagne électorale. À l'époque nous voulions concrétiser un projet en faveur d'orphelins au Maroc : lors de notre premier voyage socioculturel, j'avais repéré l'orphelinat de Tétouan. Je suis donc allée chez ce monsieur et je lui ai parlé de notre projet. Il m'a dit qu'il était lui-même orphelin (en fait c'était un mensonge, je l'ai appris plus tard: ses parents étaient seulement séparés). Il avait bien compris ma sensibilité à cette cause... Et donc j'avais mis en place avec lui, à Tanger, une structure pour impressionner le Wali pour qu'il appuie notre projet pour les orphelins. J'avais financé cette structure sur mes propres fonds; lui, il n'a jamais donné la partie qu'il devait mettre... On a donc ouvert un centre à Tanger, avec quelqu'un qui était payé par nous pour identifier toutes les associations au Maroc travaillant sur la question des orphelins. À sa suggestion nous avons engagé là-bas quelqu'un de sa famille, que j'ai payé deux ans pour ce travail. Et rien n'a été fait! Après cet échec très dur, j'ai travaillé avec Abdelmalek: j'avais compris que c'était quelqu'un qui faisait les choses et à qui on pouvait faire confiance ».

11 En 2008 il est entré au cabinet de la ministre des Affaires sociales et de la Solidarité, qui lui a demandé d'élaborer un projet national - le premier au Maroc - sur le handicap. Le projet a été approuvé et lancé. Il a déjà permis à 625 personnes en situation de handicap de trouver un emploi dans une entreprise ou une collectivité territoriale. Il dit : « Madame la Ministre voulait quelqu'un qui soit un militant, parce qu'il connaît le terrain, parce qu'il peut aider à mettre en place des règles applicables, et en même temps quelqu'un de confiance qui peut négocier, faire le lien entre les milieux politiques et la base». Le gouvernement marocain l'a choisi pour le représenter auprès des Nations Unies sur les questions de handicap. 
de certains besoins vitaux. Ce constat se fait en général à partir d'une rencontre avec une personne ressource jugée fiable (Abdelmalek A.) et capable d'être le relais de l'activité sur place à travers une association marocaine (ici La Colombe). L'objectif visé est de trouver les partenaires locaux (agents municipaux, élus) et éventuellement nationaux (ministres, personnels administratifs, entourage du roi), voire internationaux (ONG, Nations Unies), qui conduiront à terme les pouvoirs publics marocains à développer la politique publique qui fait défaut. Nous avons retrouvé ce mode opératoire dans les autres projets de Dar el Ward.

\section{Un orphelinat dans l'hôpital civil de Tétouan}

Il y a deux orphelinats à Tétouan. L'un est dirigé par une pharmacienne qui est aussi laïque et féministe. Les dirigeants de l'autre sont proches du Parti Justice et Développement, un parti islamiste engagé de longue date dans les activités caritatives et qui a gagné les élections de novembre 2011. Dar el Ward a choisi d'aider au financement de ce second orphelinat, alors qu'une ONG espagnole aidait déjà l'autre. Mais elle a aussi mis sur pied des activités communes aux deux orphelinats.

L'orphelinat jouxte l'hôpital civil. Depuis 1995, l'association y a donné des médicaments, des vêtements et des fournitures scolaires pour les « orphelins $»^{12}$. Les négociations ont été longues et difficiles avec les personnes, de tendance islamiste, qui gèrent ce lieu.

"Nous avons travaillé sept ans pour améliorer cet orphelinat. C'est né au départ de lait et de Pampers. Mais par exemple quand j'ai vu que le bâtiment était pourri, j'ai rêvé d'amener des chômeurs pour venir repeindre et faire les travaux.

J'ai négocié avec l'ONEM ${ }^{13}$ qui a débloqué beaucoup d'argent pour envoyer de jeunes chômeurs faire leur stage à Tétouan en venant reconstruire l'orphelinat. On a commencé petit, en se laissant marcher sur les pieds; et après tu peux faire ce que tu veux. Tout le bâtiment a été cassé et reconstruit. Toutes les matières premières étaient financées par Dar el Ward. L'État belge a beaucoup payé ; ils ont fait $70 \%$ du travail. Et puis le reste était à terminer. Avec le temps on a appris à négocier, à devenir exigeants. Un interlocuteur marocain a été essentiel, c'est le docteur El B. qui, à l'époque, en 2000-2001, était délégué de la santé à Tétouan ${ }^{14}$. Il nous a aidés

12 Schématiquement, le Droit musulman établit la filiation à partir du père et du père seulement. Les enfants qui naissent hors mariage, sans père identifiable, sont donc considérés comme " orphelins » et placés dans des orphelinats peu financés. Ce problème est récurrent dans tout le Maghreb ; il est lié au statut de la femme dans le Droit musulman. C'est donc la faiblesse des droits des femmes en tant que mères qui est en cause. Partout dans le monde les questions de développement humain et celles des droits des femmes sont très étroitement imbriquées. $\mathrm{Au}$ Maroc, depuis la réforme en 2004 de la Moudawana - le Code de la famille - la mère célibataire, qui jusque-là n'avait pas le droit d'élever son enfant, peut désormais demander au père de l'enfant qu'il reconnaisse sa paternité ; ou déclarer elle-même son enfant à l'état-civil si son père (à elle) accepte de lui donner son nom. Mais ces démarches demeurent très difficiles, car leur réussite est laissée au bon vouloir des juges.

13 L'équivalent en Belgique de l'ANPE en France.

14 Le docteur El B. est actuellement Directeur exécutif de l'Association Lalla Salma pour la lutte contre le Cancer que la femme du roi du Maroc, Lalla Salma, a fondée en 2006 et préside. Nous avons rencontré par la suite le docteur El B. à Rabat ; voir ci-après. 
à mettre en place des formations pour les éducatrices, avec des formateurs venus de Belgique et un système de parrainage $e^{15}$ pour la prise en charge des enfants de cet orphelinat [par des familles belgo-marocaines et belges]. Avec son aide on a organisé au sein de l'orphelinat des groupes d'enfants par âge et par activité. En Belgique j'ai cherché quelqu'un qui pourrait porter au Maroc ce projet. J'ai trouvé la femme d'un sociologue belge, d'origine marocaine, Aïcha ; elle m'a aidée dans les rapports avec les autorités marocaines. [Quand le gros du travail a été réalisé] nous avons divergé sur les méthodes, mais elle a pris la responsabilité du projet et elle a continué le travail sur place. On déclenche l'action et une fois qu'elle fonctionne par elle-même on les lâche».

Najat n'avait plus visité l'orphelinat depuis deux ans. Nous y sommes allées à l'improviste. Les éducatrices ont accueilli Najat avec ferveur. Elles m'ont confirmé l'investissement de Dar el Ward et montré l'évolution de l'orphelinat : au lieu des quatrevingts enfants présents au début de l'action il n'en reste plus que vingt-quatre. Cette diminution est due, selon elles, à une meilleure prise en charge des mères célibataires qui peuvent désormais envisager de garder leur enfant auprès d'elles, car elles reçoivent de l'aide dans leur recherche d'un logement et d'un emploi. De plus la procédure d'adoption a été facilitée.

Le lieu était parfaitement propre et rangé. Les éducatrices ont cependant dit à Najat que le problème qui restait à régler concernait les « orphelins » (enfants abandonnés) handicapés, qu'il s'avérait impossible de placer dans une famille. Or, au-delà de six ans, aucune structure n'existe pour les accueillir. Nous avons rencontré plusieurs de ces enfants handicapés, nettement plus âgés que les autres, qui grandissent là faute de mieux. Najat a aussitôt défini la situation en tant que nouveau défi à relever par son association.

\section{Un projet de soins aux enfants diabétiques de Tétouan}

Il est assez rare de tomber sur un projet transnational qui, soutenu par l'argent venu du « Nord » développé, renvoie à ce « Nord» des savoirs originaux. Tel est cependant le cas du projet portant sur les enfants diabétiques de Tétouan. Il est porté à Bruxelles par l'hôpital universitaire de la Vrije Universiteit Brussel (VUB) de Jette, dans la région bruxelloise, avec l'appoint de Dar el Ward et à Tétouan par l'association « Aide aux enfants diabétiques ». Le rôle de pivot y est tenu par Mahjouba L., née à Bruxelles de migrants marocains, éducatrice auprès d'enfants diabétiques à l'hôpital de la VUB (AZ VUB) dans le service d'un Professeur spécialiste du diabète. Pour des raisons encore mal élucidées, le diabète est relativement très fréquent au Maghreb et chez les personnes originaires du Maghreb, y compris chez les enfants et le service n'arrivait pas à stabiliser la santé des nombreux enfants diabétiques qui venaient s'y faire soigner.

15 Les fonds ont été récoltés par des donateurs et des donatrices. Parmi elles, un groupe de vingttrois mères de famille âgées de cinquante ans et plus jouent un grand rôle dans toutes les actions de solidarité de Dar el Ward. Elles récoltent des fonds, sont marraines d'enfants orphelins, etc. Sans être membres officiellement de l'association, elles forment un groupe appelé les Hajets' Club. 
C'est en 2003, alors qu'elle travaillait pour l'OCIV (Overleg Centrum Integratie van Vluchtelingen, le Centre pour l'Intégration des Réfugiés) dont la mission est d'aider les émigrés à mettre sur pied des micro-projets de production ou de service dans leur ville ou pays d'origine, que Najat avait rencontré Mahjouba qui cherchait à mieux connaître l'environnement des familles au Maroc. Elle l'avait invitée aux « ateliers participatifs » (voir infra) financés par l'OCIV, qu'organisait Abdelmalek à Tétouan. C'est ainsi que Mahjouba avait trouvé un partenaire local fiable : l'association « Aide aux enfants diabétiques » de Tétouan était entrée en contact avec deux médecins de l'hôpital de Tétouan, une femme et un homme qui travaillaient bénévolement avec cette association. Or ces médecins semblaient obtenir de bien meilleurs résultats que l'équipe belge. Celle-ci chercha à les faire venir à Bruxelles. Mahjouba organisa, avec l'aide de Dar el Ward, une première soirée de soutien pour récolter les fonds nécessaires ; ensuite c'est elle, ses amies et sa famille qui continuèrent à trouver de l'argent. Elle avait obtenu une bourse pour financer son projet et les firmes pharmaceutiques avec qui travaille l'hôpital AZ VUB acceptèrent de le sponsoriser, permettant ainsi de prendre en charge les nombreux voyages des médecins et infirmiers entre Bruxelles et Tétouan.

La pratique innovante des médecins de Tétouan consiste, pour l'essentiel, à enseigner aux enfants à se « monitorer » et à se soigner eux-mêmes. La femme médecin l'explique ainsi :

"On a réussi à faire connaître la maladie aux enfants. Trente enfants en savent plus que nous sur leur maladie. Cette automédication est nécessaire aussi pour aider les parents. Ce n'est pas seulement le matériel, l'insuline. C'est aussi comment les enfants remplissent-ils leur carnet de santé. Avant, certains mentaient mais aujourd'hui on leur donne les appareils, ils ne peuvent plus mentir. Ils vivent

beaucoup avec nous. On fait plein d'activités avec eux. Mahjouba vient chaque année. Elle a appris les habitudes alimentaires marocaines, elle a appris l'arabe.

Elle apporte avec elle les outils, les techniques. Des rencontres avec l'équipe médicale sont organisées ici et à Bruxelles ».

L'approche mise au point à Tétouan a été importée avec succès à Bruxelles ; y compris sa dimension ludique, car il s'agit d'enfants et c'est en leur montrant comment « jouer » à se « monitorer » et se soigner qu' on peut leur faire intérioriser les pratiques indispensables.

Tétouan occupe une place centrale dans la genèse des activités transnationales de l'association Dar el Ward. Toujours accomplies de façon bénévole du côté belge, celles-ci se sont multipliées dans toutes les régions du Maroc et se sont fortement diversifiées dans leurs contenus au cours des années, non seulement à Tétouan mais aussi ailleurs. Cet activisme multiforme est sous-tendu par une dynamique d'ensemble qui s'inscrit dans la durée, une éthique très solide, et une constante réflexivité collective. Najat :

« Entre 1996 et 2003, nous avons surtout travaillé activement pour répondre aux besoins matériels des différents projets. Plus de cinquante convois ont été acheminés vers le Maroc, dont Tétouan. C'est en 2003 que nous avons pensé à réaliser des ateliers participatifs. Y participaient à la fois les porteurs des projets au "Nord" (Bruxelles) et les bénéficiaires du "Sud" (au Maroc). Par exemple, à Tétouan, le projet de prise en charge des enfants abandonnés. Il a été monté par Dar El Ward et 
l'association "Pièces à conviction" sur Bruxelles. Le deuxième projet était consacré aux mères célibataires. Il était porté sur place par une infirmière sociale belgomarocaine [membre de Dar el Ward] venue s'installer à Tétouan. Le troisième projet était celui de la Colombe Blanche. Le quatrième projet portait sur la prise en charge et l'éducation des enfants diabétiques. Le partenaire local, c'est l'Association des enfants diabétiques ».

Abdelmalek A, qui organise ces ateliers, y fait participer certains des usagers des projets, « des publics cibles pris comme personnes ressources », comme dit Najat. Car c'est ainsi - telle est sa conviction et celle de l'association - que peuvent être identifiés les véritables besoins :

"Suite à ces ateliers participatifs, l’un des résultats a été la création d'un partenaire local qui avait comme mission la coordination et le suivi des différents projets mis en place sur Tétouan : le "Centre Nord Sud pour la Solidarité, le Développement et les Cuvres sociales" ".

\section{Un projet d'aide à la construction d'un puits dans un village du Haut-Atlas}

C'est Fatima Kh., l'une des fondatrices de Dar el Ward, qui est à l'initiative de ce projet $^{16}$. Sur le plan technique, le partenaire marocain est un ingénieur agronome originaire de Aït Youcef, un village à $50 \mathrm{kms}$ qui - contrairement à Inmel - se trouve au bord d'une route nationale. Inmel, quant à lui, est situé loin de cette route, au bout d'une longue piste. L'ingénieur travaille également avec d'autres ONG telles qu'OXFAM et avec une importante association de femmes immigrées de Bruxelles - francophone celle-ci -, l'association Cactus.

Nous nous sommes arrêtés à Aït Youcef pour négocier avec l'ingénieur agronome l'envoi à Inmel du groupe électrogène dont a longtemps bénéficié ce village : il n'en a plus besoin maintenant que l'électricité est arrivée. Mohamed Kh., le frère de Fatima, est un élu de la Région de Meknès-Tafilalet ; il est aussi le partenaire marocain du projet sur le puits d'Inmel. Najat :

"Il nous a facilité toute la partie administrative, autorisation, etc., ainsi que la prise en charge de la construction du château d'eau, et la programmation de l'eau et de l'électricité pour ce village par la Région. Il a aussi facilité l'arrivée et la distribution de convois humanitaires dans quatre villages, dont Aï Youcef et Inmel ».

16 Elle a une histoire migratoire particulière où s'exprime sa force de caractère. L'aînée d'une famille de Meknès, elle était partie seule à dix-neuf ans à Bruxelles, en 1969, pour y travailler comme domestique à demeure pour un médecin. Elle n'avait pas le droit de sortir ; elle travaillait énormément ; l'arrangement était que son salaire serait versé chaque mois à sa famille. Un jour elle s'est rendue compte qu'ils n'avaient jamais rien reçu. Elle s'est alors enfuie et a déposé plainte avec l'aide du Mouvement contre le Racisme, l'Antisémitisme et la Xénophobie (MRAX). Elle a eu gain de cause. Le MRAX l'a ensuite aidée à obtenir un diplôme d'aide familiale aux seniors. Parallèlement, elle s'était fortement engagée dans les mouvements sociaux d'étudiants marocains. Son mari aussi est très actif dans l'association. 
Le budget nécessaire à la construction d'un puits et d'un poulailler communal à Inmel se partage en deux : 13000 euros collectés par Dar el Ward sous forme de dons, 13000 euros attribués par la Région de Meknès-Tafilalet. Une association de retraités de Meknès a accepté de superviser les travaux. Le puits a été creusé, un château d'eau a été construit, ainsi que des conduites d'eau jusqu'à la mosquée et à la fontaine. On n'attend plus que le groupe électrogène pour pomper l'eau. L'initiative de Dar el Ward a attiré l'attention des autorités régionales sur ce village : depuis que le puits a été creusé il est question d'y amener l'électricité17. Sur ce dernier exemple, on voit qu'ici aussi les actions menées sur une base transnationale ont un effet de levier (ou de catalyseur) qui démultiplie leurs effets.

\section{À Rabat, un projet de soins aux enfants leucémiques}

Ce projet a été lancé en 2005 à l'initiative de Fatima El B., qui est membre de Dar el Ward depuis treize ans. Fatima avait toujours vécu à Rabat jusqu'à ce que sa fille soit victime d'un grave problème au rein. On avait d'abord tenté de la sauver à l'hôpital pour enfants de Rabat. Mais les médecins, devant leur impuissance, lui avaient alors suggéré d'emmener sa fille à Bruxelles pour y faire suivre un traitement spécifique.

«Ma fille devait être transplantée; mais malheureusement elle est morte. Je suis restée à Bruxelles ; mais depuis, j'ai eu envie de m'engager pour aider des familles d'enfants malades. C'est à cause de ça que j'ai été vers Dar el Ward».

À côté de son activité bénévole, Fatima enseigne l'arabe et fait de nombreux voyages entre Bruxelles et Rabat. Elle y reste en contact avec le Dr Mohamed Kh., qui était à la tête du service d'oncologie de l'hôpital pour enfants et se plaignait amèrement de ne pouvoir apporter à ses petits patients venus de tout le Maroc, les médicaments nécessaires pour soigner correctement leur leucémie.

Un partenariat s'est mis en place petit à petit entre ce médecin marocain et l'association. Des collectes de fonds ont été organisées par Dar el Ward à Bruxelles pour récolter l'argent nécessaire à l'achat et à l'acheminement des médicaments indispensables. Sur place, à Rabat, c'est Kenza, une jeune animatrice marocaine sœur de Fatima que l'association a choisie pour assumer la responsabilité du projet.

Kenza est d'abord venue informellement, de manière régulière, animer la salle d'attente en jouant avec les enfants. Elle prenait ainsi contact avec leurs familles. L'objectif de son action était, et est toujours, de connaître leurs besoins réels et de " trouver les moyens d'y répondre pour qu'elles n'abandonnent pas les soins $\gg^{18}$. Depuis quelques mois Kenza est officiellement rémunérée par Dar el Ward qui a également financé et installé une salle de jeu. Kenza :

"Les enfants arrivent en courant vers moi. J'ouvre la salle, j'organise des tours de rôle, je leur donne les jouets qu'ils veulent. J'ai pas pu jouer quand j'étais

17 Actuellement seul le tiers du territoire du Maroc est couvert en matière d'infrastructures pour l'eau et l'électricité.

18 On retrouve ici ce que les services sociaux appellent en France l'« approche globale ». 


\begin{abstract}
petite, alors là je joue. Avant d'ouvrir la salle, j'ai fait le tour des parents pour voir $s$ 'ils avaient besoin de quelque chose. Ils m'expliquent la situation de santé de leur enfant, mais aussi leurs autres soucis, par exemple les difficultés qu'ils rencontrent pour arriver jusqu'à Rabat. Ils viennent de toutes les régions du Maroc. Ils sont adressés par les médecins de leur région vers Rabat. Parfois je les oriente vers

le service social de l'hôpital, qui peut les aider financièrement par exemple pour acheter les médicaments à $30 \%$ du prix. Ce sont des donateurs qui permettent cela, mais encore faut-il les trouver $\gg$.
\end{abstract}

Nous avons accompagné Najat à l'hôpital pour enfants de Rabat. Dans la salle d'attente, une vive discussion était engagée entre les parents présents. Certains d'entre eux s'interrogeaient sur le nouveau système d'assistance aux malades en difficulté, le RAMED (Régime d'Assistance Médicale aux Plus Démunis) qui est en train d'être mis en place. En principe il a pour but de soigner gratuitement, près de chez elles, les personnes qui ne peuvent pas payer le coût des soins. Mais s'il n'y a pas le service adéquat là où elles habitent et qu'elles vont jusqu'à Rabat pour soigner leur enfant, l'administration de l'hôpital leur signifie qu'elles devront payer elles-mêmes le traitement ; l'enveloppe de soins est définie région par région. Un vent de panique parcourt l'assistance. On est en décembre 2011 et le nouveau système est censé entrer en vigueur en janvier 2012. La discussion en est là quand le Dr Mohammed Kh. vient nous chercher. Il est aussitôt assailli de questions sur ce sujet par les parents présents. Il nous dira ensuite qu'il est effectivement très préoccupé lui aussi. Comment soigner quand le pronostic vital est engagé mais qu'il faut d'abord arriver à lever les freins administratifs mis par l'organisation hospitalière à l'exercice de son métier ? À propos de Najat il dit : «Je la suivrai jusqu'au bout du monde! ». Il fait ensuite un bilan de leur collaboration :

«J'ai rencontré Fatima, Najat et leur association en 2005 quand j'étais confronté à un grave problème. Pour soigner les enfants atteints de cancer, nous avions besoin de disposer d'un médicament que les firmes marocaines ne voulaient pas produire, car elles considéraient que ce n'était pas rentable. Il s'agissait de l'Actinomycine D. Il fallait absolument le faire venir de l'étranger. Entre 2005 et aujourd'hui, c'est grâce au travail de lobbying auprès des firmes pharmaceutiques et de récolte de fonds de Najat et de son association que nous les avons obtenus. J'ai une grande nouvelle: depuis quelques mois nous n'avons plus besoin de cette aide, car le relais a été pris par la Fondation Lalla Salma de lutte contre le cancer.

Depuis 2005, nous avons aussi monté un vaste programme d'échanges avec le service du professeur Éric S., cancérologue spécialiste des leucémies de l'hôpital Reine Fabiola de l'Université Libre de Bruxelles. Nous avons établi un protocole de prise en charge de la leucémie, le meilleur du Maghreb : 70 à $80 \%$ de guérisons, comme en Europe, grâce à un suivi personnalisé de chaque patient pour qu'il ou elle n'abandonne pas son traitement. Dar el Ward, avec Fatima, Kenza et Najat, nous ont aidés à améliorer le service et la vie des patients. Elles sont venues avec des jeunes de Bruxelles qui ont repeint les murs, fait des dessins, mis des rideaux dans les chambres, mis des séparations entre les boxes où sont examinés les petits malades de mon service ».

Tout en parlant le Dr Kh. nous fait visiter son service. Soudain Najat s'exclame : elle a remarqué que les rideaux et les séparations ont été à nouveau refaits. Les travaux ont été effectués par la Fondation Lalla Salma et la Reine en personne est venue les inspecter. 
Le Dr Kh. termine la visite en parlant avec émotion de cette petite fille atteinte d'un cancer du foie pour laquelle il fallait absolument procéder à une greffe venant du foie de sa mère. Ses jours étaient comptés et la greffe s'avérait impossible à financer. C'est alors qu'en deux semaines elle a pu être sauvée, grâce à une campagne de collecte de fonds de Dar el Ward et à l'accord d'un chirurgien flamand, qui avait accepté de négocier avec son hôpital la baisse du coût de l'intervention.

Des descriptions des quatre projets précédents, se dégage un schéma récurrent des étapes mises en œuvre par l'association pour construire un projet. Cela commence par le repérage, par l'une des membres de Dar el Ward, d'un besoin vital non satisfait auquel il faut répondre le plus vite possible et parfois de manière urgente. La personne qui a identifié le problème sera celle qui va suivre directement le déroulement du projet au Maroc et faire le lien avec les partenaires à Bruxelles : par exemple les Services de la Coopération et du Développement de la Région Flamande, des donateurs privés, des médecins le cas échéant, des écoles professionnelles qui recrutent dans la région de Bruxelles des jeunes gens qui viendront faire des travaux de réfection. La responsable du projet cherche au Maroc une personne de confiance qui pourra porter concrètement le projet. Elle y cherche aussi des personnes-ressources disposant de moyens d'action, et qui pourraient à terme faire prendre aux autorités marocaines le relais de l'action entreprise.

C'est par exemple le cas du docteur El B., qui vient de l'hôpital de Tétouan et qui est devenu le Directeur exécutif de la Fondation Lalla Salma. Il était ce jour-là dans le service d'oncologie de l'hôpital. C'est lui qui a organisé récemment l'achat et l'approvisionnement en médicaments de ce service et qui a poursuivi les travaux débutés à l'initiative de Dar el Ward. Il décrit ainsi la politique suivie par la Fondation :

"Nous avons une vision nationale sur la santé. La Fondation élabore un plan national de lutte contre le cancer pour les dix ans à venir. C'est un plan qui permet de normaliser et standardiser les dépenses. On ne construit pas un monstre pour répondre aux besoins de la population. On part des idées et des actions d'associations comme Dar el Ward : elles jouent un rôle de catalyseur, de liaison, comme dans une réaction chimique. Najat et les autres sont venues avec des idées précises.

Après quelque temps nous les avons mises en œuvre sur une plus grande échelle

(...). La fondation Lalla Selma est elle-même une courroie de transmission entre les hôpitaux et les ministères $\gg$.

\section{DISCUSSION}

Une étude de cas ne permet pas la généralisation. Cependant elle permet la " contre-généralisation » (Bertaux, 2010) dans la mesure où elle contredit une de ces fausses généralisations par lesquelles procède toujours le sens commun. Un seul cas, une seule étude de cas y suffit.

En l'occurrence nous avons voulu vérifier s'il était exact que les activités transnationales sont essentiellement portées par des hommes. Quand elles le sont par des femmes, nous disait la littérature, ce serait exclusivement par des femmes en tant que mères, dans le cadre de formes transnationales de famille. 
Nous étions sceptiques, car nous avions déjà rencontré, au cours d'une recherche antérieure, deux exemples d'activité transnationale d'associations d'immigrées (Delcroix et al., 1996, 2010a). Elles mobilisaient toutes deux des femmes originaires de divers pays ; l'une concernait l'aide aux enfants vivant dans un orphelinat à Constantine, l'autre l'information juridique des femmes et des filles dans une ville du Nord-Ouest du Maroc. Étaient-ce là des exceptions?

Nous sommes à la recherche du phénomène soi-disant inexistant, non seulement pour nous assurer de son existence mais pour commencer à en dessiner les caractéristiques. Le choix de Bruxelles et de sa situation quasi-expérimentale ouvrait la voie à l'étude d'une hypothèse supplémentaire : le communautarisme qu'encourage le modèle multiculturel du côté flamand, par la promotion du maintien des liens « culturels » avec le pays d'origine, facilite-t-il le développement d'activités transnationales ; tandis que l'assimilationnisme prôné du côté francophone le freinerait? Il est trop tôt pour répondre. Le fait que Dar el Ward soit animée par des Marocaines socialisées du côté flamand va dans le sens de l'hypothèse, ainsi que la fierté patriotique et les motivations religieuses qui inspirent leurs activités humanitaires transnationales. Mais des associations francophones s'activent aussi de leur côté.

En tout état de cause ces activités existent : nous les avons rencontrées. Peut-on en dire un peu plus? Plusieurs des caractéristiques les plus saillantes des activités transnationales de Dar el Ward semblent former un système cohérent entre elles mais aussi avec le fait qu'elles sont initiées par des associations de femmes.

Ce qui frappe c'est d'abord la proximité de ces femmes (et des hommes qu'elles ont cooptés en fonction de leurs valeurs) avec des personnes rendues vulnérables par leur situation d'enfant abandonné, de mère célibataire, de handicapé ou de malade. Cette proximité leur permet d'identifier des besoins impératifs auxquels il faut répondre dans l'urgence.

Elles/ils auraient pu en rester là et inscrire ces activités bénévoles dans l'humanitaire, le caritatif, l'aide ponctuelle apolitique sans vision élargie. Mais tel n'est pas le cas : on observe de façon récurrente un mode opératoire très professionnel qui met en cohérence les actions menées à Bruxelles et au Maroc. On part de la personne en situation de besoin vital ; on concentre l'attention sur elle parce qu'elle sait mieux que quiconque ce qui lui manque, aussi parce qu'elle n'est pas seulement objet de l'aide mais qu'elle l'informe, la guide, y participe en coopérant (c'est ce que font par exemple les enfants diabétiques). Il s'agit aussi de faire remonter les besoins à une tout autre échelle « spatiale », l'association recherche des partenaires privés et institutionnels, en Belgique et au Maroc. L'objectif visé est, in fine, de transmettre la responsabilité et le suivi de l'action entreprise, et d'en commencer une autre; car il y a tant à faire.

Les actions de l'association parviennent aussi à s'inscrire dans différentes échelles temporelles. Si la capacité à répondre à une situation d'urgence reste prioritaire, la recherche de la durabilité des projets est cruciale et favorise la réflexion et l'action stratégiques. 
Dans chaque projet la compréhension du contexte, des enjeux, la recherche d'actrices/acteurs locaux de confiance, la mobilisation de ressources, impliquent un haut degré de réflexivité. Plus l'action entreprise au niveau local répond avec précision et efficacité aux besoins vitaux réels, plus il y a de chances que le relais en soit pris - plus ou moins rapidement - par une institution officielle. C'est ainsi, par la qualité reconnue de ses actions bien plus que par le lobbying, que cette association participe à la construction de politiques publiques.

Les activités transnationales poursuivant des objectifs économiques - et peut-être aussi politiques - cherchent à promouvoir les intérêts, personnels ou collectifs, de ceux qui les portent. Nous n'avons rien observé de tel à Dar el Ward, bien au contraire : la détermination des responsables à ne jamais se rémunérer, à toujours agir de façon bénévole, est frappante. Tout au moins dans cette association. Au rebours de l'esprit utilitariste, il s'agit donc ici de solidarité désintéressée.

Est-ce en rapport avec le fait que l'association a été fondée et est dirigée par des femmes? Le terme de care est tendanciellement associé à la féminité, non seulement parce que ce sont des femmes qui y ont été assignées depuis des siècles, mais en raison de travaux récents. L'étude qui a lancé les recherches sur le care (Gilligan, 1984) a remis en lumière l'hypothèse d'une affinité profonde entre, d'une part, les chemins différents suivis par la construction progressive des fondements de l'identité genrée. La mère est une femme, la petite fille aussi, elle peut donc grandir tout en restant dans la relation fusionnelle. Le petit garçon, au contraire, aura besoin pour se développer de s'affirmer comme différent de sa mère : elle c'est elle, mais moi c'est moi. D'où l'hypothèse de Gilligan : devenues adultes les femmes auraient plutôt tendance à développer une orientation vers autrui, tandis que chez les hommes l'individualisme dominerait. Souci des autres, opposé au souci de soi ; d'un côté on perçoit le monde en termes de relations interpersonnelles ; de l'autre on se pense en son centre. Don de soi et care d'une part, affirmation agressive de ses propres intérêts de l'autre. Bien entendu on force ici le trait. Carol Gilligan ne va pas jusqu'à affirmer que la dichotomie serait radicale, et il n'est d'ailleurs pas difficile d'évoquer des exemples d'hommes profondément altruistes et de femmes profondément intéressées. Mais en termes de tendances, d'orientations profondes des valeurs, cette théorie n'est pas dépourvue d'intérêt.

Elle semble en tous cas utile pour analyser les activités transnationales dans une perspective de genre. Cependant nous ne pensons pas que le terme de care leur convienne. Nous lui préférons le terme de développement humain créé en 1990 par Mahbub ul Haq et Amartya Sen au sein du Programme des Nations Unies pour le Développement (PNUD). Il inclut toute une série d'activités : soins médicaux, enseignement (scolarisation des filles en particulier), prise en charge des personnes dépendantes, dont le point commun est de contribuer à maintenir en vie, à instruire, à remettre sur pied et à favoriser le développement physique et psychique, corporel et culturel des citoyens d'un pays. Le PNUD publie chaque année un classement des pays selon leur " Indice de Développement Humain »; ce qui les différencie n'est pas tant l'existence ou l'inexistence de tels ou tels services (santé, éducation, etc.), mais la mesure dans laquelle les citoyens y ont accès indépendamment de leur revenu. À cet égard le Maroc a longtemps été fort mal classé. 
Mais il se trouve que ce pays est en train de se transformer. Le roi Mohammed VI, qui avec son entourage a longtemps concentré l'essentiel des pouvoirs d'État, a lancé depuis 2006 de vastes réformes au sein d'une grande Initiative Nationale pour le Développement Humain, cofinancée d'ailleurs par le PNUD. Alors que les divers « projets » initiés par Dar el Ward avaient tous commencé, comme il se doit, à l'échelle locale - bien que certains aient commencé à essaimer vers des niveaux plus élevés - voici que, d'en haut, un programme national vient en quelque sorte à leur rencontre.

Or, l'un des principaux problèmes des politiques et des experts qui mettent en place les nombreuses réformes en cours (celle du système de santé par exemple), c'est de n'avoir qu'une vision d'ensemble du pays qui devient de plus en plus floue au fur et à mesure que l'on descend du niveau national au régional - déjà bien différencié - puis au subrégional, et enfin au niveau local. À ce dernier niveau, ce sont les acteurs locaux qui possèdent les savoirs et les savoir-faire, les connaissances et les compétences indispensables. Aussi les associations implantées de longue date et dont l'efficacité et la qualité de l'action a été reconnue dans la durée figurent parmi les partenaires de premier choix.

On discerne ici les indices d'une logique profonde à l'œuvre : logiques structurelles de situations, logiques d' " action située » récurrentes, logiques de réseaux, logiques contextuelles. Il faudra bien plus qu'une étude de cas pour en dégager les grandes lignes que nous n'avons fait ici qu'esquisser.

Mais nous avons au moins répondu à la question de départ : les femmes émigrées/ immigrées développent-elles aussi, en tant que femmes (et non en tant que mères), des activités transnationales ? Nous avons choisi de chercher la réponse à partir des Marocaines de Bruxelles en raison de l'aspect quasi-expérimental du contexte bruxellois. Mais nous n'imaginions nullement que nous allions trouver si facilement, chez une association de Marocaines de Bruxelles, une telle intensité d'activités transnationales. Et nous savons déjà qu'elle n'est pas la seule dans ce cas, loin de là.

Il existe de par le monde d'innombrables « communautés immigrées », constituées pour près de la moitié par des femmes. La logique nous conduit à poser l'hypothèse qu'il doit en être pour elles peu ou prou de même que pour ces Marocaines ; car l'hypothèse inverse serait que nous serions tombées par hasard sur la seule exception.

Il est plausible que plus est fort et patent le différentiel entre politiques publiques de développement humain du pays d'origine et du pays d'accueil, plus vraisemblable toutes choses égales par ailleurs - est l'apparition d'un « courant » d'activités transnationales en sens inverse de l'émigration.

Il ne serait donc pas étonnant que les recherches futures montrent que quel que soit le flux migratoire, des femmes émigrées/immigrées - voire leurs filles - cherchent à sauver des vies d'enfants ou de jeunes femmes dans leur pays d'origine ; qu'elles s'associent pour y parvenir et qu'elles y trouvent, en raison de leurs convictions humanistes, patriotiques, religieuses ou autres, suffisamment de gratifications personnelles pour continuer et amplifier leur action. On pourrait même découvrir que leur action finit parfois par conduire l'État du pays concerné lui-même à prendre enfin les responsabilités de politique publique qui lui incombent. 


\section{Références bibliographiques}

ADAM Ilke (2010) Au-delà des modèles nationaux d'intégration. Analyse des politiques d'intégration des personnes issues de l'immigration des entités fédérées belge, Thèse de Doctorat en sciences politiques, Université Libre de Bruxelles.

BERTAUX Daniel (2010) Les récits de vie, Paris, Armand Colin, 128 p.

DELCROIX Catherine (2010a) Prévenir et résoudre les conflits : deux générations de médiatrices socioculturelles en action, in Philippe Hamman, Maurice Blanc, Jean-Yves Causer Dirs., Ville, environnement et transaction démocratique, Bruxelles, Peter Lang, pp. 93-110.

DELCROIX Catherine (2010b) S'engager dans la durée. De la relation d'enquête aux effets de la publication, in Jean-Paul Payet et al., La relation d'enquête. La sociologie au défi des acteurs faibles, Rennes, Presses Universitaires de Rennes, pp. 131-142.

DELCROIX Catherine (2007) Ressources subjectives et construction d'un capital d'expérience biographique : l'exemple des médiatrices socio-culturelles, in Claudine Dardy et Cédric Frétigbé, L'expérience professionnelle et personnelle en questions, Paris, L'Harmattan, pp. 83-116.

DELCROIX Catherine, BESKI Chahla, RADJA MATHIEU Zaïda et BERTAUX Sandrine (1996) Médiatrices dans les quartiers fragilisés : le lien, Paris, La Documentation Française, 136 p.

EGGERICKX Thierry, BAHRI Amel, PERRIN Nicolas et WETS Johan (2010) La migration internationale et la population d'origine étrangère en Belgique, Bruxelles, DGSIE, 185 p.

FIBBI Rosita et d'AMATO Gianni (2008) Transnationalisme des migrants en Europe : une preuve par les faits, Revue Européenne des Migrations Internationales, 24 (2), pp. 7-22.

GAUDIER Jean-Pierre et Philippe HERMANS (1991) Des Belges marocains, Bruxelles, De Boeck, $230 \mathrm{p}$.

GEERTZ Clifford (1973) The Interpretation of Cultures, New York, Basic Books, $480 \mathrm{p}$.

GILLIGAN Carol (1982) In a Different Voice, Cambridge, Harvard University Press, 184 p.

GLICK SCHILLER Nina, BASH Linda and BLANC-SZANTON Cristina (1992) Transnationalism: A New Analytic Framework for Understanding Migration, Annals of the New York Academy of Science, 645, pp. 1-24.

LACROIX Thomas, SALL Leyla et SALZBRUNN Monika (2008) Marocains et Sénégalais de France : permanences et évolution des relations transnationales, Revue Européenne des Migrations Internationales, 24 (2), pp. 23-41.

MARTINIELLO Marco et BOUSETTA Hassan (2008) Les pratiques transnationales des immigrés chinois et marocains de Belgique, Revue Européenne des Migrations Internationales, 24 (2), pp. 45-66.

PESSAR Patricia and MAHLER Sarah J. (2003) Transnational Migration: Bringing Gender in, International Migration Review, 37 (3), pp. 812-846.

PORTES Alejandro (1999) Conclusion: Towards a new world - the origin and the effects of transnational activities, Ethnic and Racial Studies, 22 (2), pp. 463-477.

PORTES Alejandro (1997) Globalization from Below: The Rise of Transnational Communities, Princeton, Princeton University Press, $27 \mathrm{p}$.

REA Andrea (2003) Les politiques publiques et les associations immigrées, in Alberto Gabbiadini, Marco Martiniello et Jean-François Potelle Éds., Politiques d'immigration et d'intégration : de l'Union européenne à la Wallonie, Namur, Institut Jules-Destrée, pp. 189-223.

REA Andrea, JACOBS Dirk et TERNEY Cécile (2010) Les comportements électoraux des minorités ethniques à Bruxelles, Revue Française de Sciences Politiques, 60 (4), pp. 691-717.

VEITH Blandine (2005) Engagement associatif et individuation des femmes migrantes, Revue Européenne des Migrations Internationales, (21) 3, pp. 53-67. 


\title{
Les activités transnationales des femmes immigrées. L'exemple d'une association de Marocaines de Bruxelles
}

\author{
Catherine Delcroix et Daniel Bertaux
}

Les études empiriques sur les activités transnationales des immigrés, qu'elles soient de type économique, politique ou socioculturel, tendent à montrer qu'elles sont surtout portées par des hommes. Cela signifie-t-il que les émigrées n'en auraient pas ? Une enquête sur les associations de Marocaines de Bruxelles montre que plusieurs d'entre elles ont des activités transnationales parfois très développées. Nous en présentons ici une étude de cas d'une association qui a créé en vingt ans plus d'une vingtaine de projets toujours actifs dans différentes régions du Maroc. Elle y vient en aide à des personnes en situation de vulnérabilité : handicap physique, enfants abandonnés, diabétiques ou leucémiques, mères célibataires, etc. Il s'agit d'activités humanitaires de développement humain, accomplies avec professionnalisme, et dont l'État marocain commence à s'inspirer pour ses réformes des politiques publiques.

\section{Transnational Activities of Migrant Women. The Case of a Moroccan Association in Brussels}

\author{
Catherine Delcroix and Daniel Bertaux
}

Empirical studies on transnational activities focused on economic, political or sociocultural goals tend to show they are mostly carried out by men. Does that mean that migrant women have none? An on-going research project on non-profit associations of Moroccan women in Brussels shows that some of them have long developed transnational activities. One case study will be presented. In twenty years this association has created about twenty "projects" in various parts of Morocco, all still in operation. They concern persons in situations of vulnerability: abandoned children, children suffering from diabetes, from leukaemia, in situation of physical handicap; or single mothers, etc. These activities of human development are achieved with great professionalism. It is now recognized that they may help fine-tuning some of the major reforms of public policies (healthcare, welfare, handicap, women's rights, etc.) which are presently being designed in Morocco.

\section{Las actividades transnacionales de las mujeres. El caso de una asociación de mujeres marroquíes en Bruselas}

\author{
Catherine Delcroix y Daniel Bertaux
}

Los estudios empíricos sobre las actividades transnacionales de los inmigrantes, tanto si sonde tipo económico, político o sociocultural tienden a mostrar que son llevadas a cabo sobretodo por hombres. ¿Significa esto que no hay mujeres emigrantes? Una encuesta sobre las asociaciones de mujeres marroquíes en Bruselas muestra que algunas de ellas tienen actividades transnacionales a veces muy desarrolladas. Presentamos aquí un estudio de caso. Esta asociación creó hace veinte años más de veinte proyectos que siguen estando activas en distintas regiones de Marruecos. Acude en ayuda de personas en situaciones de vulnerabilidad: niños abandonados, diabéticos, leucémicos, madres solteras, disminuidos físicos... Se trata de actividades humanitarias, de cuidados, de desarrollo humano, llevadas a cabo con todo el profesionalismo necesario y del que el estado marroquí ha comenzado a inspirarse para sus reformas de políticas públicas. 